\title{
The Predictors of Anxiety and Depression in Head and Neck Cancer Patients
}

\author{
Yen-Ju, Chen
}

Da-Yeh University, Department of Nursing, Changhua, Taiwan R.O.C..

\section{Introduction}

- Head and neck cancer (HNC) is one of the most common cancer in the worldwide, and the fourth rank of top ten common cancer of male in Taiwan.

- HNC patients suffer from physical and psychological dysfunction from cancer diagnosis. However, previous studies indicated that anxiety and depression significantly impacted on patients' mental health and quality of life.

\section{Objectives}

Therefore, the purposes of the study are to explore:

- the severity of anxiety and depression;

- the predictors of anxiety and depression.

\section{Method}

- The study was a cross sectional study with consecutive sampling.

- Patients were assessed by structured questionnaires, including

- Hospital Anxiety and Depression Scale (HADS)

- Symptom Severity Scale (SSS)

- Background information after treatment

- Data was analyzed by descriptive statistics and logistic regression.

\section{Results}

- In a total of 108 patients, the severity of psychological distress was mild at average, but around $32 \%$ of patients were identified anxiety and depression.

- Marital status, tumor stage, continued alcohol, tobacco, and betel nut used, and symptom severity were significantly predicted to anxiety.

- Gender, marital status, tumor stage, continued alcohol, tobacco, and betel nut used were significantly predicted to depression.

Table 2. Severity of anxiety and depression

\begin{tabular}{lc}
\hline Variables & N (\%) \\
\hline Anxiety & \\
$\quad$ Clinical cases $(\geqq 11)$ & $\mathbf{3 5}(\mathbf{3 2 . 2})$ \\
$\quad$ Borderline cases $(8-10)$ & $7(6.7)$ \\
Non-cases $(0-7)$ & $66(61.1)$ \\
Depression & \\
Clinical cases ( $\geqq 11)$ & $\mathbf{3 5 ( 3 2 . 2 )}$ \\
Borderline cases $(8-10)$ & $3(2.7)$ \\
Non-cases $(0-7)$ & $70(64.8)$ \\
\hline
\end{tabular}

Table 1. Demographics and disease information

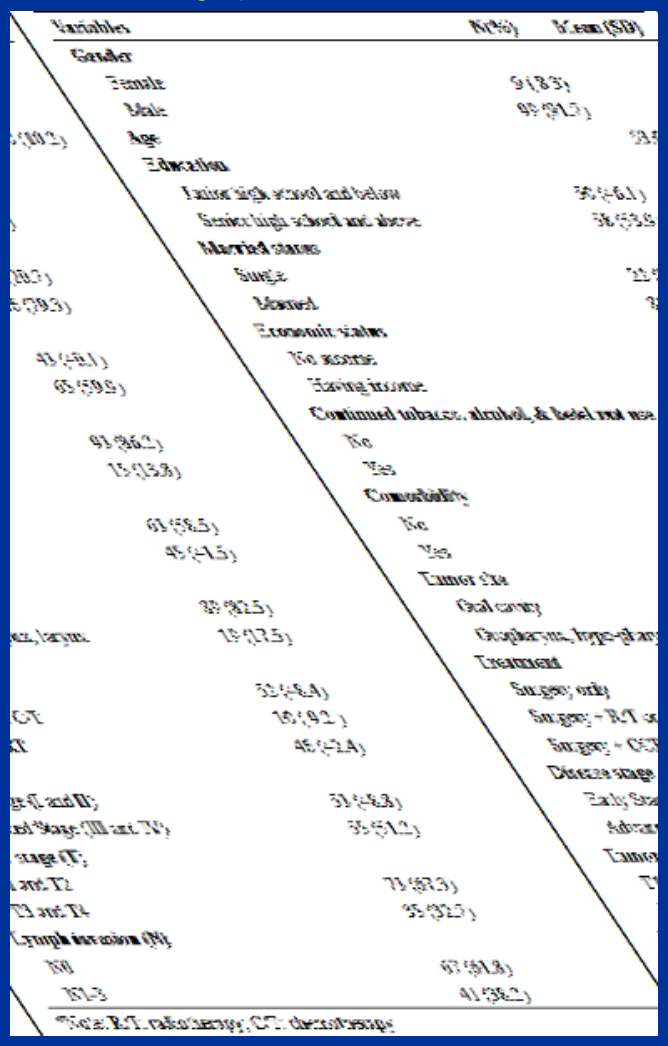

Table 3. Predictors of anxiety and depression

\begin{tabular}{|c|c|c|c|c|c|}
\hline \multirow{2}{*}{ Variables } & \multirow{2}{*}{ B estimate (SE) } & \multirow{2}{*}{$\begin{array}{c}\text { Odds } \\
\text { ratio }\end{array}$} & \multicolumn{2}{|c|}{$95 \% \mathrm{CI}$} & \multirow{2}{*}{$P$ value } \\
\hline & & & Lower & Upper & \\
\hline \multicolumn{6}{|l|}{ Anxiety predictors } \\
\hline Constant & $-6.89(1.31)$ & & & & 0.000 \\
\hline Male & $-1.47(0.79)$ & 0.23 & 0.05 & 1.08 & 0.075 \\
\hline Disease stage: $\mathrm{T} 3$ and $\mathrm{T} 4$ & $-1.36(0.42)$ & 0.26 & 0.11 & 0.58 & $0.002 * *$ \\
\hline Continued tobacco, alcohol, \& betel nut use & $2.36(0.77)$ & 10.56 & 2.32 & 48.11 & $0.002 * *$ \\
\hline Education level below junior high school & $0.32(0.35)$ & 1.85 & 0.93 & 3.71 & 0.081 \\
\hline Marital status: single & $0.93(0.41)$ & 2.53 & 1.13 & 5.62 & $0.023^{*}$ \\
\hline Having income & $0.67(0.36)$ & 1.95 & 0.96 & 3.96 & 0.065 \\
\hline Symptom severity & $0.02(0.01)$ & 1.02 & 1.00 & 1.03 & $0.020^{*}$ \\
\hline \multicolumn{6}{|l|}{ Depression predictors } \\
\hline Constant & $-6.07(1.24)$ & & & & 0.000 \\
\hline Male & $1.62(0.79)$ & 5.04 & 1.07 & 23.61 & $0.040^{*}$ \\
\hline Disease stage: $\mathrm{T} 3$ and $\mathrm{T} 4$ & $1.29(0.43)$ & 3.65 & 1.59 & 8.38 & $0.002^{* * *}$ \\
\hline Continued tobacco, alcohol, \& betel nut use & $2.21(0.76)$ & 9.09 & 2.03 & 40.67 & $0.004 * *$ \\
\hline Education level below junior high school & $0.58(0.34)$ & 1.78 & 0.91 & 3.46 & 0.090 \\
\hline Marital status: single & $0.91(0.40)$ & 2.48 & 1.13 & 5.42 & $0.023^{*}$ \\
\hline
\end{tabular}

\section{Conclusions}

- HNC patients suffered from anxiety and depression after cancer treatment. The screening and assessment of anxiety and depression should be earlier and routinely.

- Future studies may provide mental support or referral service to improve their mental health. 